\title{
Metodologias qualitativa e visual no estudo da parentalidade digital: Desafios metodológicos
}

\author{
Alexandra Maria Barradas Batista ${ }^{1}$ e Rosalina Pisco Costa ${ }^{2}$ \\ 1 Universidade de Évora, Portugal | abbatista@uevora.pt; alexandra.mbb@gmail.com | \\ https://orcid.org/0000-0002-2486-508X \\ 2 Universidade de Évora e CICS.NOVA, Portugal | rosalina@uevora.pt | \\ https://orcid.org/0000-0003-4549-9012
}

\begin{abstract}
Resumo: Este texto antecipa e reflete criticamente sobre um conjunto de obstáculos de natureza metodológica e epistemológica no contexto mais amplo de um estudo qualitativo destinado a compreender o modo como os usos que pais e filhos fazem da tecnologia de informação e comunicação (TIC) enformam a construção social em torno da parentalidade competente. Inspirada pela generalização recente de dispositivos tecnológicos e de software, que possibilitam e facilitam a comunicação à distância, esta investigação visa a desocultação da experiência da parentalidade digital, numa abordagem qualitativa, intensiva e em profundidade. A recolha de dados será efetuada através de entrevistas semiestruturadas e histórias familiares (episódios), de forma a que as pessoas entrevistadas partilhem com a investigadora diferentes segmentos de interação em rede (e.g.: WhatsApp, Facebook) que ajudem a ilustrar, expandir ou contrapor as experiências narradas como socialmente significativas.
\end{abstract}

Palavras-chave: Cultura Popular; Métodos; Relações Familiares; Socialização; Tecnologias da Informação e Comunicação.

Qualitative and Visual Methodologies in the Study of Digital Parenting: Methodological challenges

\begin{abstract}
This text anticipates and critically reflects upon a set of a methodological and epistemological obstacles in the broader context of a research project aimed at understanding how the uses that parents and children make of information and communication technology (ICT) shape the social construction around competent parenting. Inspired by the recent widespread use of technological devices and software, which enable and facilitate communication at a distance, this research aims to unveil the experience of digital parenting, in a qualitative, intensive, and in-depth approach. Through semi-structured interviews and collecting family stories (episodes), participants will be invited to share with the researcher different segments of network interaction (e.g.: WhatsApp, Facebook), that help to illustrate, expand or counter the experiences narrated as socially significant.
\end{abstract}

Keywords: Popular Culture; Methods; Family Relationships; Socialization; Information and Communication Technologies.

\section{Introdução e Contextualização}

Numa época em que se verifica a utilização massificada dos dispositivos tecnológicos e de software, muitos são os desafios que se colocam à sociedade em geral e às famílias em particular. Sociologicamente ancorada, propomo-nos desenvolver uma investigação qualitativa para estudar a parentalidade digital com alusão à prática de sharenting ${ }^{1}$.

A partilha de conteúdos nos media sociais é abundante, segue diversos objetivos e formas de comunicação, nomeadamente a partilha de vídeos, imagens e mensagens escritas. $\mathrm{Na}$ atualidade, os pais tendem a partilhar vários conteúdos, tais como fotografias ou vídeos dos seus filhos online com diversos fins, sejam eles para mostrar à família que está mais longe ou para mostrar aos pares que são "bons pais".

\footnotetext{
${ }^{1}$ Este artigo parte da reflexão sobre uma investigação, em curso, financiada pela Fundação para a Ciência e Tecnologia I.P. (FCT, MCTES, FSE, UE), através da concessão de uma bolsa (Ref. 2020. 06828.BD), para a obtenção do Doutoramento em Sociologia.
} 
Afinal, os media sociais, oferecem aos seus utilizadores uma oportunidade de comunicar com um número elevado de pessoas sem ter de investir muito tempo e muito esforço (Brosch, 2018; Blum-Ross \& Livingstone, 2017; Damkjaer, 2018; Lim, 2018; Lipu \& Siibak, 2019; Steinberg, 2020).

A parentalidade competente é um conceito emergente em ciências sociais, particularmente em sociologia. Insiste na ideia de que aos pais contemporâneos não basta que sejam pais, impõe-se que sejam "bons pais" (Miller, 2005, 2011, 2017). Isto faz com que um conjunto grande de prescrições normativas - e respetivos mecanismos de vigilância - se imponham aos pais com força coerciva e "normalizadora" no modo como a contemporaneidade constrói socialmente e parentalidade. Tais orientações incidem sobre um sem número de dimensões da vida quotidiana das relações que envolvem pais e filhos, seja no domínio da educação, dos valores e da socialização em termos gerais, seja no domínio do vestuário, da alimentação, do consumo ou do lazer. O domínio dos usos e significados associados às tecnologias de informação e comunicação (TIC) e presença em redes sociais virtuais não constitui exceção.

Em plataformas de natureza mais privada, isto é, as que se restringem a pequenos grupos de natureza familiar ou amical, como o WhatsApp, seja em redes de perfil mais aberto, como o Facebook, muitos pais começam, desde muito cedo, a partilhar online conteúdos sobre os filhos (sharenting). Inclusivamente antes do nascimento, aquando da partilha online de ecografias, os pais contribuem para alimentar a pegada digital das crianças (Damkjaer, 2018; Leaver, 2015). Até que ponto são os pais "bons pais" quando empreendem tais partilhas? Estarão eles a respeitar os direitos das crianças? Importa, pois, adotar um olhar mais global e aprofundado sobre a parentalidade em contexto digital. Como se consubstancia? Que propósitos serve? Quais as perceções a respeito por parte de pais e filhos? Em suma, de que modo é que os usos que pais e filhos fazem da tecnologia de comunicação enformam a construção social em torno da parentalidade competente na contemporaneidade?

A resposta ao conjunto de questões aqui levantadas permitirá iluminar áreas de sombra na sociologia portuguesa e contribuir para o debate informado sobre potencialidades e limites da parentalidade digital e sua relação com a proteção dos direitos das crianças (ONU, 1989). O objetivo geral da investigação é compreender o modo como os usos que pais e filhos fazem da tecnologia de informação e comunicação enformam a construção social em torno da parentalidade competente na contemporaneidade. De modo detalhado, importa desocultar as estratégias práticas e discursivas implementadas através das tecnologias de informação e comunicação pelos pais em nome da garantia de segurança, bem-estar e felicidade dos filhos, para, a partir daí, compreender como essas estratégias são experienciadas e contestadas na tensão gerada entre os sentidos da parentalidade competente e os direitos das crianças.

\section{Metodologia, Epistemologia e Ética}

\subsection{Olhar por dentro a parentalidade digital}

Propomo-nos desenvolver um estudo qualitativo, intensivo e em profundidade, operacionalizado por meio de um desenho de investigação dinâmico, aberto aos contributos que advêm quer da teoria, quer da evidência empírica (Dezin \& Lincoln, 2000; Mason, 2002). Procuramos compreender as perspetivas dos indivíduos, neste caso, dos diferentes elementos das famílias (pais e filhos), através das suas experiências, opiniões, perceções e significados acerca da partilha de conteúdos dos filhos, pelos pais, online, isto é, como é que estes entendem subjetivamente a sua realidade (Sampieri et. al, 2010; Kornblit, 2007).

Com enfoque na experiência digital, pretendemos conhecer as perceções das famílias 'a partir de dentro' (Mason, 2002) sobre as estratégias práticas e discursivas implementadas através da tecnologia de informação e comunicação, pelos pais, em nome da garantia da segurança, bem-estar e felicidade dos filhos. 
Paralelamente, intentamos compreender como é que estas estratégias são experienciadas e contestadas na tensão gerada entre os sentidos da parentalidade competente e os direitos das crianças. Consideramos que a metodologia qualitativa se revela como a mais adequada para a prossecução da presente investigação, pois privilegia o «contexto da descoberta» como ponto de partida da investigação, através da observação do sujeito e da sociedade em interação, bem como dos factos e das emoções que os corroboram (Guerra, 2006). Através desta metodologia podemos explorar diversas dimensões do mundo social, incluindo a vida quotidiana, perceções, experiências e representações dos participantes, bem como os processos sociais, instituições, discursos, significados e o funcionamento das relações que mantêm uns com os outros (Manson, 2002). Este tipo de metodologia (qualitativa) baseia-se num processo indutivo (Guerra, 2006) e não deve limitar-se a descrever os fenómenos que estão a ser estudados, deve também contribuir para a produção de teorias, tendo por base o que chamamos de Grounded Theory, isto é, a criação de teoria a partir dos dados (Glaser \& Strauss, 1967; Guerra, 2006).

\subsection{Protocolo de Recolha, Tratamento e Análise de Dados}

Prevê-se que a seleção da amostra seja realizada de forma intencional e eventualmente em 'bola de neve' (Flick, 2013). Adicionalmente, será constituída por 'casos múltiplos', isto é, famílias (pais/mães/filhos/as) com diferentes características (capital escolar, capital económico, composição, morfologia etc.) e por contraste-aprofundamento, pois pretendese efetuar comparações em profundidade intra e inter-casos, de forma a explorar pistas que marquem as diferenças e semelhanças entre eles (Guerra, 2006).

Como unidade de análise, serão consideradas crianças entre os 10 e os 18 anos de idade, bem como díades parentais (compostas por um pai/mãe e um filho/a) incluídos em morfologias familiares diversificadas (e.g.: nucleares, monoparentais, recompostas), em fases distintas da carreira parental (e.g.: filhos pequenos, filhos adolescentes), e de dimensão e composição variável da fratria (e.g.: famílias de filho único e mais filhos, idade, género, relação de parentesco, nomeadamente irmãos biológicos, "meios-irmãos").

As entrevistas centrar-se-ão sobre a recolha de narrativas orais em torno da experiência da parentalidade, fazendo uso de entrevistas de episódio (Flick, 2005), tendo em vista a recolha de histórias familiares sobre acontecimentos ('episódios') considerados significativos pelos sujeitos da observação em torno das principais dimensões em estudo: práticas, contextos e sentidos da parentalidade digital. No decorrer da entrevista as pessoas entrevistadas (pais - adultos e filhos - crianças) serão interpeladas a partilhar com a entrevistadora segmentos de interação em rede (e.g.: WhatsApp, Facebook) que ajudem a ilustrar, expandir ou contrapor as narrativas obtidas. Os dados recolhidos serão analisados mediante técnicas qualitativas de análise de conteúdo, temática, formal e estrutural (Bardin, 1977; Krippendorff, 2003), inspiradas pelas orientações metodológicas subjacentes à análise de narrativas visuais (Margolis \& Pauwels, 2011) e etnografia digital (Pink et al, 2015).

\subsection{Desafios ao Estudo da Parentalidade Digital em Perspetiva Qualitativa}

Nesta secção pretendemos antecipar e refletir criticamente sobre um conjunto de obstáculos de natureza metodológica e epistemológica que prevemos encontrar durante a concretização do presente estudo: ao nível do recrutamento de participantes, ao nível da recolha, análise e interpretação de resultados de dados e ao nível do compromisso ético transversal. No momento em que nos encontramos, e atendendo à vocação do Congresso Ibero-Americano em Investigação Qualitativa que serve de enquadramento a esta reflexão, certar-nos-emos de forma mais demorada sobre a recolha, análise e interpretação de resultados de dados. 
Ao nível do recrutamento de participantes, o facto de trabalharmos com famílias e crianças constitui desde logo um desafio, dado o carácter privado do tema, pois nem sempre as pessoas estão dispostas a partilhar as suas experiências com um desconhecido (Sampier et al, 2010). O facto de nesta investigação estarem previstas entrevistas a crianças parece adensar esta questão, pois a literatura evidencia que as crianças muitas vezes mostram sinais de fadiga após 15/20 minutos de entrevista; que pode ser difícil captar a sua atenção para o assunto em estudo; e que também os pais parecem genericamente pouco recetíveis à entrevista aos filhos (Saramago, 2001). Além disto, a solicitação do consentimento informado (Flick, 2013), quer aos pais, quer às crianças, constitui um desafio adicional (Almeida \& Delicado, 2017), na medida em que da obtenção deste, e no quadro do Regulamento Geral sobre a Proteção dos Dados (RGPD) atualmente em vigor (União Europeia, 2016), em Portugal segundo a lei n.ำ 58/2019 publicada em Diário da República n. - 151/2019, Série I de 2019-08-08, sempre depende a investigação para avançar. Por último, o estado atual de pandemia em que nos encontramos (e que se prevê prolongar no futuro próximo), também se constitui como um desafio no acesso ao campo, tanto no contacto com possíveis entrevistados, como na realização das entrevistas, as quais implicarão a proximidade suficiente para permitir a visualização por parte da entrevistadora de materiais partilhados entre pais e filhos através de plataformas como o Facebook ou WhatsApp.

Ao nível da análise, tratamento e interpretação de resultados, os desafios multiplicam-se. O facto de esta investigação se desenrolar num intervalo temporal limitado, de apenas três anos, e com a responsabilidade de cumprir o cronograma pré-estabelecido por ser um projeto financiado pela Fundação para Ciência e Tecnologia para a obtenção do grau de doutor, faz com se procure a maximização do trabalho intensivo que caracteriza o ofício do investigador qualitativo por meio da triangulação de dados (Denzin \& Lincoln, 2000). Todavia, esta opção acarreta maior morosidade no que se refere à recolha, tratamento $e$ análise de dados (Costa, 2011, 2019). Um outro desafio será o facto de os entrevistados terem experiências diferentes da investigadora principal, por exemplo no que se refere ao exercício da parentalidade, naquilo que consideram ou não ser um 'bom pai/mãe' ou na forma como utilizam as TIC. Para lidar com estes obstáculos propõe-se uma planificação detalhada e atempada das atividades, bem como a adesão estrita aos códigos deontológicos que enformam a prática da investigação científica.

A integração de metodologias visuais (Pink et al., 2015) no decorrer da entrevista constitui porventura o principal desafio da investigação ora apresentada. As pessoas entrevistadas serão chamadas a partilhar com a investigadora diversos segmentos de interação em rede que sejam considerados significativos na explicitação das experiências narradas (e.g. fotografias, vídeos, diálogos ou comentários em Facebook ou WhatsApp). Deste modo, quer adultos, quer crianças, farão uso dos seus computadores pessoais, smartphones ou tablets para "mostrar" à investigadora, eventualmente também "apontar" e "destacar" partes desses segmentos. Esta metodologia, que podemos designar de criativa (Kara, 2015), na medida em que conjuga de forma nova e orientada às questões e objetivos de investigação técnicas de recolha de dados pré-existentes (e.g.: entrevista e elicitação visual) permite aceder a diferentes dimensões da vida das pessoas, obtendo retratos mais finos e ricos do seu quotidiano. Porém, exigirá necessariamente um aprofundamento de competências da parte da investigadora principal nesta matéria, obrigando-a a 'mergulhar' num domínio relativamente novo, em expansão e evolução permanente.

A utilização de metodologias visuais torna mais premente a abordagem dinâmica e nãolinear que a investigação qualitativa coloca sobre as fases da recolha, análise de dados e interpretação de resultados. Para lidar com este desafio propomo-nos explorar de forma intensiva o modelo interativo de análise de dados qualitativos tal qual preconizado por Miles, Huberman \& Saldaña (2014). Este modelo articula de forma dinâmica as componentes de recolha de dados, apresentação de dados (data display), redução de informação e extração e verificação de conclusões. 
A utilização de metodologias visuais enquanto instrumento de recolha de dados permitirá captar formas de expressão não-verbais, estimulando uma maior participação e envolvimento dos entrevistados, assegurando uma relação menos assimétrica entre o investigador e entrevistado e implicando um maior enfoque na recolha de dados, que não centrada exclusivamente na oralidade. O modo como os recursos eletrónicos (e.g.: computador pessoal, smartphone ou tablet) são mobilizados no discurso, mas também como os conteúdos a que através deles acedemos são expostos e representados, tanto na sua apropriação, quanto na sua contestação por parte das pessoas entrevistadas, serão igualmente alvo de análise qualitativa de conteúdo. O facto de virmos a trabalhar com crianças evidencia ainda mais a importância da utilização destes métodos, pois estes estimulam o envolvimento das crianças (Almeida et al., 2012), cuja participação é mais limitada quando recorremos a metodologias baseadas exclusivamente na oralidade (Graue \& Walsh, 2003).

Porque as metodologias qualitativas são na sua génese flexíveis e multidimensionais, equacionamos algumas soluções que minimizem os obstáculos que daí advêm nas fases de recolha, tratamento e análise de dados. Em concreto, aquando da recolha de dados prevemos a negociação do consentimento informado tendo em vista o registo vídeo da entrevista, inclusive nos casos em que as entrevistas decorram online (e.g.: via Zoom ou Skype). Adicionalmente, prevemos o recurso a software de análise qualitativa de dados (e.g.: NVIVO, @ QSR International) como forma de auxiliar o manuseamento e tratamento multimétodo da informação recolhida.

Como os exemplos anteriores patenteiam, ao nível ético e de modo transversal, vários desafios se colocam. Desde logo, o direito a ser informado sobre os objetivos da investigação, o princípio da voluntariedade de participação e o direito à confidencialidade e ao anonimato dos dados, a que o investigador deve estar sempre atento à medida que avança com a investigação (APS, 1992), são desafios que se afiguram particularmente relevantes na medida em que pretendemos estudar em simultâneo adultos e crianças. Ademais, olhar a materiais que os primeiros partilham online sobre os segundos é também trazer para a análise a rede pessoal maior em que uns e outros se incluem, seja a família alargada, seja a rede de pares conhecidos e desconhecidos de adultos e crianças (Almeida \& Delicado, 2017).

\section{Conclusões}

A metodologia qualitativa que subjaz ao desenho da investigação em curso pretende, em última instância, obter informações sobre as perceções, experiências e sentidos atribuídos à parentalidade digital, quer do ponto de vista dos pais (pai e mãe), quer do ponto de vista das crianças. Aliar as entrevistas à recolha de material visual (e.g.: fotografias, vídeos) permite obter resultados mais ricos e matizados sobre as experiências dos vários protagonistas com as TIC e também conhecer as complexas interceções entre os media, cultura, social e material (Pink et al., 2015).

Discutir, refletir e antever potenciais obstáculos e desafios de natureza metodológica, epistemológica e ética suscitados pela investigação qualitativa constitui um desafio tão importante quanto necessário na investigação científica em geral, e qualitativa em particular, pois isso significa a preparação e mobilização de soluções reflexivamente ajustadas, logo, mais eficientes porque menos apoiadas na decisão episódica e improvisação casuística. 


\section{Referências}

Almeida, A.; Carvalho, D.; Delicado, A. \& Alves, N. (2012). Pela mão das crianças: metodologias em construção. VII Congresso Português de Sociologia, Sociedade, crise e reconfigurações.

Almeida, A. N. \& Delicado, A. (2017). Crianças online. Metodologias visuais, novas descobertas e desafios éticos. In Ferreira, S. V. (org.), Pesquisar Jovens: caminhos e desafios metodológicos (pp. 135-161). Lisboa: ICS - Imprensa de Ciências Sociais.

APS (1992). Código Deontológico - Associação Portuguesa de Sociologia. Lisboa: Associação Portuguesa de Sociologia.

Bardin, L. (1977). Análise de conteúdo. Lisboa: Edições 70.

Blum-Ross, A. \& Livingstone, S. (2017). Sharenting: parent blogging and the boundaries of the digital self. Popular Communication. The International Journal of Media and Culture. V. 15 (2). Pp. 110-125.

Brosch, A. (2018). Sharenting - Why do parents violate their children's privacy?, pp. 75-85, The New Educational Review.

Costa, R. (2011). Ridendo Castigat Mores. A transcrição de entrevistas e a (re) construção social da realidade. VIII Congresso Português de Sociologia, 40 anos de demorcracias: progressos, contradições e prospetivas.

Costa, R. (2019). iPhone, iResearch. Exploring the Use of Smart Phones in the Teaching and Learning of Visual Qualitative Methodologies. Journal of Visual Literacy, 38 (1-2), 153-162. [DOI: https://doi.org/10.1080/1051144X.2019.1567073].

Damkjaer, M. S. (2018). Sharenting = Good Parenting? Four Parental Approaches to Sharenting on Facebook p. 209-218 in Giovanna Mascheroni, Cristina Ponte \& Ana Jorge (eds.) Digital Parenting. The Challenges for Families in the Digital Age. Göteborg: Nordicom.

Denzin, N. K. \& Lincoln, Y. (eds.) (2000). Handbook of Qualitative Research, 2nd ed.. Thousand Oaks: Sage Publications.

Flick, U. (2015). Métodos Qualitativos na Investigação Científica. Lisboa: Monitor.

Flick, U. (2013). Introdução à Metodologia de Pesquisa. Porto Alegre: PENSO Editora.

Glaser, B. G., \& Strauss, A. L. (1967). The Discovery of Grounded Theory: Strategies for Qualitative Research. Chicago: Aldine Publishing Company.

Graue, E., \& Walsh, D. (2003). Investigação etnográfica com crianças: teorias, métodos e ética. Lisboa: Fundação Calouste Gulbenkian.

Guerra, I. C. (2006). Pesquisa Qualitativa e Análise de Conteúdo. Sentidos e formas de uso. Estoril: Princípia.

Kara, Helen. 2015. Creative Research Methods in the Social Sciences: A Practical Guide. Bristol: Policy Press.

Kornblit L. A. (2007). Metodologías cualitativas en ciencias sociales. Buenos Aires: Editorial Biblos.

Krippendorff, K. Content Analysis: An Introduction to Its Methodology. 2. ed. Beverly Hill, CA: Sage Publications, 2003.

Leaver, T. (2015). Born Digital? Presence, Privacy, and Intimate Surveillance. In Hartley, John \& W Qu (Eds.), Re-Orientation: Translingual Transcultural Transmedia. Studies in narrative, language, identity, and knowledge (pp. 149-160). Shanghai: Fudan University Press.

Lim, S. S. (2018). Transcendent Parenting in Digitally Connected Families. When the Technological Meets the Social. In Giovanna Mascheroni, Cristina Ponte \& Ana Jorge (eds.) Digital Parenting. The Challenges for Families in the Digital Age (pp. 31-39). Göteborg: Nordicom.

Lipu, M. \& Siibak, A. (2019). 'Take it down!': Estonian Parents' and pre-teens' opinions and experiences with sharenting., SAGE Journals: Media International Australia, Vol.170(I), pp.57-67. 
Margolis, E., \& Pauwels, L. (eds.) (2011). The SAGE Handbook of Visual Research Methods. London, Sage.

Mason, J. (2002). Qualitative Researching, (2nd Ed.) London: Sage Publications.

Miles, Matthew. B., Michal A. Huberman, e Johnny Saldaña. 2014. Qualitative Data Analysis: A Methods Sourcebook. 3ª edição. Los Angeles: SAGE.

Miller, T. (2005). Making Sense of Motherhood. A Narrative Approach. Cambridge: Cambridge University Press.

Miller, T. (2011). Making Sense of Fatherhood. Gender, Caring and Work. Cambridge: Cambridge University Press.

Miller, T. (2017). Making Sense of Parenthood. Caring, Gender and Family Lives. Cambridge: Cambridge University Press.

Pink. S.; Horst, H.; Postill, J.; Hjorth, L.; Lewis, T. \& Tacchi, J. (2015). Digital ethnographyPrinciples and Pratice. SAGE PUBLICATIONS.

Punch, F K. (1998). Introduction to Social Research. London, SAGE Publications

Sampieri, H.; Collado, C. \& Lucio, P. (2010). El proceso de la investigación cualitativa. Parte 3, (pp.361-542). México: McGraw-Hill/Interamericana Editores, S.A de C.V.

Sampieri, H.; Collado, C. \& Lucio, P. (2010a). Los enfoques cuantitativo y cualitativo en la investigación científica. In Metodología de la Investigación. (5. 르. Ed.) (pp. 1-32). México: McGraw Hill.

Saramago, S. (2001). Metodologias de Pesquisa Empírica com crianças. Sociologia, Problemas e Práticas, n.ำ 35, pp. 9-29.

Steinberg, S. (2020). Growing up shared: how parents can share smarter on social media and what you can do to keep your family safe in no-privacy world. Naperville: Sourcebooks.

União Europeia (2016). Regulamento Geral de Proteção dos dados (RGPD). [Disponível em url: https://eur-lex.europa.eu/legal-content/PT/TXT/?uri=celex\%3A32016R0679] 